\title{
Children's Psychological Reactions to the COVID-19 Pandemic
}

\author{
Betty Pfefferbaum ${ }^{1}$
}

Accepted: 29 August 2021 / Published online: 6 October 2021

(c) The Author(s), under exclusive licence to Springer Science+Business Media, LLC, part of Springer Nature 2021

\begin{abstract}
Purpose of Review This paper reviews the literature on the psychological effects of the COVID-19 pandemic on children and the reactions of vulnerable children.

Recent Findings Research reveals increases in clinically significant depression, suicidal ideation and behavior, and some anxiety symptoms. Substance use studies suggest an inadvertent decrease in substance use in some youth though findings are inconsistent across substances and for males and females. Children with pre-existing emotional and behavioral problems are especially vulnerable though some children appear to improve in the context of public health measures which have decreased the stresses associated with school and socialization. In addition, children with pre-existing problems are likely to have established resources and relationships that may protect them relative to other children.

Summary COVID-19 has had a major effect on the mental health of children around the world, but findings should be considered preliminary until more rigorous research has been conducted.
\end{abstract}

Keywords Child disaster mental health $\cdot$ COVID-19 $\cdot$ Disaster $\cdot$ Disaster reactions $\cdot$ Pandemic $\cdot$ Vulnerable children

\section{Introduction}

The World Health Organization declared COVID-19 a global pandemic on March 11, 2020. Despite a rich literature on children's reactions to disasters and the fact that, historically, pandemics have been among the most devastating of disasters [1], the psychosocial effects of prior pandemics have not been well studied in children [2]. After briefly considering the novel issues raised by COVID-19, the epidemiology of the disease in children, and overarching issues, this paper describes research methods in the extant literature, reviews the emerging literature on the psychological effects of the pandemic on children and the reactions of children with pre-existing emotional and behavioral vulnerabilities, and discusses findings in the context of the larger literature on children's disaster reactions. The paper also identifies limitations in the extant research and suggests issues for future investigation.

This article is part of the Topical Collection on Child and Family Disaster Psychiatry

Betty Pfefferbaum

Betty-Pfefferbaum@ouhsc.edu

1 Department of Psychiatry and Behavioral Sciences, College of Medicine University of Oklahoma Health Sciences Center, 920 Stanton L. Young Boulevard, WP3217, P.O. Box 26901, Oklahoma City, OK 73126-0901, USA

\section{Novel Issues Raised by COVID-19}

Numerous differences between the COVID-19 pandemic and more common natural and manmade events include its worldwide reach and protracted threat; the invisible nature of the danger with many people unaware of their own personal exposure to, or infection with, the virus until or unless they experience symptoms; the varied and uncertain prognosis of the disease; the massive death toll; the personal and global social and economic repercussions; the extensive workforce implications; and the political climate that has jeopardized the response. The need for accurate information is vast, and COVID-19 has compelled public health measures that have added unfamiliar burdens and generated reactions not typically considered in child disaster research.

\section{The Epidemiology of COVID-19 in Children}

Reports on the susceptibility of children to COVID-19 infection are inconsistent. Some contend that while children may manifest milder symptoms and lower mortality rates than adults, they are as likely to contract the disease, and they may transmit the virus [3]. A recent review and meta-analysis suggested that young children (aged 10 to 14 years and younger) are less susceptible to and may be less likely to 
transmit the disease [4]. The need for ongoing research on this issue is clear, especially in light of evolving evidence, concern about new variants of the virus and the possible increased vulnerability of children to these variants, the delay in offering vaccines to children, the resultant stress on the pediatric healthcare system and workforce, and changes in public health containment measures.

\section{Overarching Issues}

Because wide-reaching public health containment measures (e.g., school closure, home confinement) were mandated in the early months of the pandemic, the study of children's reactions cannot be considered separate from these efforts. The containment measures interfered with children's education; restricted their social interaction; curtailed their participation in usual activities, recreation, play, and extracurricular programs; and altered family dynamics. School closure interrupted numerous school-based social, health, and mental health services with potential adverse academic, health, emotional, behavioral, family, and social consequences.

Children's reactions to COVID-19, like reactions to other disasters, depend in part on their personal experiences. For example, children may contract the virus or be affected through the involvement of family members or significant others who, for example, contract the disease or participate in the essential workforce. All children, including those in the general community and the larger society, are influenced by the experiences and reactions of their families and of the community and society, by public health measures, by media coverage, and by the availability of services.

\section{Research Methods in the Extant Literature}

Extensive research has used various approaches to examine psychological reactions to the COVID-19 pandemic in children. Some studies have used routinely collected data on children's mental health and on mental health service utilization to ascertain pandemic-related changes. Most of the research has assessed universal (e.g., general population, school-based) samples rather than samples of children selected because of their particular personal involvement (e.g., children who have tested positive for the disease and/ or suffered the illness, children who know those with the disease, children of essential workers) despite the fact that many children are likely to be greatly affected by these more personal experiences. For example, using mortality and fertility data from 21 countries, modeling analysis estimated that, from March 2020 through April 2021, over one million children worldwide experienced the death of a primary caregiver (parent or custodial grandparent) [5]. Only one known study examined the psychological reactions of a sample of children who contracted the disease [6]. In 2021, Liu and colleagues [6] assessed children (aged 5 to 18 years) from Wuhan, China, who had been hospitalized for COVID-19 infection. Other studies have assessed at-risk children using general population-based and clinical samples and samples of children with pre-existing psychological vulnerabilities. Cross-sectional investigations predominate with few studies using longitudinal design to assess representative samples.

\section{Psychological Reactions}

As with disasters in general, children's reactions to COVID19 range from distress to psychiatric symptoms and disorders. The most commonly studied psychological reactions to the pandemic are discussed below.

\section{Distress}

Most child COVID-19 studies have explored specific emotional and behavioral outcomes rather than psychological distress, a common disaster reaction in children. In March 2020 (during school closure), Qin and colleagues [7] fielded a large study of a stratified cluster random sample of primary and secondary students in a Chinese province where COVID-19 rates were high. A total of $10.5 \%$ of the participants reported psychological distress, which the authors considered a high rate of distress [7] though much of the extant child disaster research considers distress to be more pervasive. The study by Qin's team [7] was conducted relatively early during the pandemic; thus, it is possible that distress increased over time, especially given that study participants were from a region with high infection rates.

\section{Probable Mental Disorder}

Some studies have considered global measures of psychological outcome. For example, using surveys of children and young people in England, Vizard and colleagues [8] compared trends in mental health in 2017 and 2020. Rates of probable mental disorder in children (aged 5 to 16 years) in $2020(16.0 \%)$ increased relative to rates in 2017 (10.8\%). Compared to those without a probable mental disorder, children (aged 11 to 16 years) and young people (aged 16 to 22 years) with a probable mental disorder were more likely to experience anxiety about the pandemic (e.g., afraid to leave home, worried that family or friends would contract COVID-19, worried about transmitting the disease to others) [8]. While not using a representative sample, a study of Bangladesh children (aged 4 to 17 years) revealed a significantly greater retrospectively reported prevalence of any predictive psychiatric disorders, emotional disorders, conduct 
disorders, and hyperactivity during home confinement relative to 6 months prior to home confinement [9].

\section{Posttraumatic Stress Disorder}

While posttraumatic stress disorder (PTSD) and posttraumatic stress reactions are commonly assessed in child disaster research, relatively few empirical child studies have examined PTSD in children in the context of the COVID19 pandemic. In the 2021 investigation by Liu and colleagues [6], 18.4\% of children who had been hospitalized for COVID-19 experienced clinically significant PTSD, but this study assessed a small convenience sample that may not be representative of all children with COVID-19. A study conducted 2 months after widespread lockdown in Saudi Arabia assessed PTSD in a random national sample of children (7 to 18 years of age) in which COVID-19 illness of the child or close relative and COVID-19 death of a close relative or friend were reported by $3.7 \%$ and $3.9 \%$, respectively [10]. Potential PTSD was identified in $13.0 \%$ of the sample [10]. In a longitudinal population-based investigation in the United Kingdom (U.K.), Wright and colleagues [11•] revealed increased PTSD symptoms 3 months after distancing measures were initiated relative to an earlier assessment from December to March 2020. In an investigation using parent report, 20.66\% of children (aged 7 to 15 years) from regions across China scored above the study's cutoff for clinical PTSD [12]. Yue and colleagues [13] found high risk for PTSD in $3.16 \%$ of their sample of Chinese primary-school aged children in a study conducted early in the pandemic (February 2020) in an area that was not severely affected by the pandemic. PTSD rates in some of these studies may have been inflated because PTSD symptoms were not clearly linked to the children's COVID-19 experiences and not all of their COVID-19 experiences would have qualified as trauma exposure for a diagnosis of PTSD.

\section{Depression and Anxiety}

A literature review revealed a number of psychological outcomes, especially depression and anxiety symptoms, in children in the context of the pandemic [14]. Symptom rates have varied greatly across studies which is not surprising given differences in samples and sampling methods, timing of assessment, and research designs used. The 2021 study of Wuhan children hospitalized for COVID-19 infection found significant symptoms of depression and of anxiety in $15.8 \%$ and $31.6 \%$, respectively [6]. The rate of depression was less than the rate in primary school students in Wuhan, but anxiety was nearly double the community rate [6]. Most studies have examined children in universal samples with some comparing rates to pre-COVID-19 levels or to general population levels. In a recent meta-analysis of general population studies during the pandemic, Racine and colleagues [15••] found clinically elevated depression and anxiety symptom rates of $25.2 \%$ and $20.5 \%$, respectively. These rates are approximately twice the rates in pre-pandemic research [15••].

Analyzing composite data from 12 longitudinal studies (10 USA, 1 Netherlands, 1 Peru), all of which conducted at least one assessment prior to the pandemic, Barendse and colleagues [16•] found a significant increase in depression symptoms, but contrary to the meta-analysis by Racine and colleagues $[15 \bullet \bullet]$, they found no change in anxiety symptoms in participants (aged 9 to 18 years) during the pandemic. Barendse and colleagues [16•] speculated that the finding of no change in anxiety symptoms overall may have obscured possible changes in some types of anxiety. For example, social interactions and social pressure may have decreased with pandemic-related physical distancing measures possibly resulting in a decline in social anxiety while other anxiety symptoms may have increased [16•].

\section{Suicidal Ideation and Behavior}

Research in the United States (U.S.) has used routine surveillance and screening data to examine rates of suicidal ideation and behavior. For example, National Syndromic Surveillance Program (NSSP) data on patient encounters across health care settings revealed an initial decrease in emergency department (ED) visits for suspected suicide attempts among adolescents and young adults (12 to 25 years of age) during the spring of 2020 in comparison to visits in the spring of 2019 , but ED visits for mental health issues and for suspected suicide attempts increased in adolescents (aged 12 to 17 years) by early May 2020 [17••]. The increase in visits for suspected suicide attempts was especially alarming in adolescent girls. Visits for adolescent boys remained stable during this period relative to 2019 data. It is noteworthy that the observed increased suicide risk for girls did not extend to suicide deaths $[17 \bullet \bullet]$.

Results of routine systematic screening (using an evidence-based tool) for suicide risk conducted with youth (aged 11 to 21 years) in a Texas pediatric ED in the U.S. between January and July revealed higher rates of suicide attempts in February, March, April, and July 2020 relative to 2019 rates for the same months [18]. Higher rates of suicidal ideation and behavior appeared to occur during months when COVID-19 stressors and public health measures intensified. All ED visits decreased during this period suggesting that only the most severe cases may have presented for care; thus, the rates of suicide-related visits may not represent true population rates [18].

Studies outside the U.S. also point to concern about suicidal ideation and behavior. For example, over one-third (36.9\%) of participants in a study of a regionally representative sample of Austrian high school students (14 to 20 years 
of age) reported suicidal ideation within the last 2 weeks during an extended period of physical distancing [19]. A longitudinal cohort study of a large sample of Chinese children initially assessed before the pandemic in a region considered at low risk for COVID-19 also found increases in non-suicidal self-injury and suicidal ideation and behavior during the pandemic [20].

Only one known study examined rates of completed suicide in children and adolescents during the pandemic. A Japanese publication reporting public suicide statistics from January 2018 to May 2020 demonstrated no significant change in rates in children and adolescents during the first wave of the pandemic when schools were closed [21]. Additional research will be needed to clarify the extent to which suicidal ideation and behavior have led to completed suicide.

\section{Behavior Problems}

A variety of behavior problems have been assessed in child COVID-19 studies. For example, a prospective study of a large national sample of parents of Japanese children (grades 1 to 12) [22] found higher clinical levels of emotional symptoms, conduct problems, hyperactivity/inattention, problems with peer relationships, and lack of prosocial behavior in May 2020 relative to levels 2 months earlier. In their population-based investigation in the U.K., Wright and colleagues [11] reported increased symptoms of disruptive behavior in 11- to 12-year-old children, especially those without prior externalizing symptoms, 3 months after the implementation of social distancing measures relative to symptoms assessed immediately before the lockdown.

\section{Substance Use and Abuse}

Youth substance use is a major public health problem worldwide [23]. A variety of factors during the pandemic may have altered substance use patterns in youth. These include heightened personal and family stress, limited social interaction, altered daily routine, relaxed structure and supervision, change in access to various products, and modifications in the delivery of substance abuse services. It is interesting that some COVID-19 investigations have documented no change or a decrease in substance use during the pandemic though findings have been inconsistent across substances and for males and females. For example, a national longitudinal study of Icelandic adolescents revealed decreased cigarette smoking, e-cigarette use, and alcohol intoxication in 15- to 18-year-old participants during the pandemic relative to rates in 2016 and 2018 [24•]. In the U.S., a prospective study of Northern California public high school students found no change in the use of tobacco-other than e-cigarettes - or in alcohol or cannabis use and a decrease in e-cigarette use (that appeared to be independent of COVID19 as the decrease began before the pandemic) before and 6 months after implementation of a state-wide stay-at-home order [25]. A national U.S. study conducted in May 2020 revealed a change in adolescent and young adult e-cigarette (including nicotine and/or other substances) use since the beginning of the pandemic [26]. With respect to nicotine e-cigarettes, almost one-third of the sample discontinued use, over one-third decreased use, and almost $20 \%$ increased use while less than $10 \%$ increased cannabis e-cigarette use and less than $10 \%$ substituted other nicotine or cannabis products [26]. Unexpected decreases in substance use may be due to reduced peer pressure and increased parental supervision and monitoring associated with home confinement. In a study of adolescents from Ontario, Canada, who retrospectively reported substance use behaviors before and after social distancing measures were implemented, Dumas and colleagues [27] found no change in the percent of adolescents using alcohol but an increase in the frequency of alcohol and cannabis use in those who were using before the pandemic. Thus, while frequency is an important measure of substance use, other dimensions such as volume, duration, and intensity may result in different findings about changes in substance use [28] and are of interest in addressing the needs of individual youth. With respect to context, Dumas and colleagues [27] found that solitary use was most common, but many adolescents used substances with peers during face-to-face and electronic communication, and some used substances in the presence of their parents.

\section{Somatic Symptoms}

Somatization is of particular interest in a medical disaster like the pandemic. A study using cluster sampling of Chinese primary school students conducted in February and March 2020 found that the incidence of somatic symptoms was $2.39 \%$ with all cases mild [29]. This seemingly low level of somatic symptoms is in contrast to rates in Chinese college students (34.85\%) assessed as part of the same study [29] and in pediatric primary care settings (between 25 and $50 \%$ ) as assessed in other studies [30].

\section{Adjustment and Resilience}

While research has focused predominantly on adverse outcomes, children's adjustment is an important consideration. In a poll of a representative sample of parents in the U.S., conducted in June 2020, approximately $14 \%$ reported worsening of their children's behavioral health since March 2020, but most parents reported no change in their children's behavioral, mental, and physical health $[31 \bullet]$. 


\section{Psychologically Vulnerable Children}

Some children have been particularly vulnerable to the pandemic, the ensuing mitigation measures, and the interruption in their daily routine and health and mental health care. A growing literature has addressed the demographic, social, and economic conditions that increase risk for negative outcome including adversity in the form of poverty, food insecurity, and detrimental living situations (e.g., homelessness, crowded living environments, inadequate sanitation); pre-existing and comorbid medical and mental health problems and physical and cognitive disabilities; disparity in, and limited access to, social and health and mental health care services; and other problems. Many of the psychosocial vulnerabilities overlap with racial/ethnic disparities to further increase risk and affect access to services for racial/ethnic minority children. The interruption of supportive services in educational, social, health, and mental health programs during mandatory containment measures likely created increased stress in families and exacerbated emotional distress and behavioral problems in vulnerable children. Of concern for this review is the vulnerability associated with pre-existing emotional and behavioral problems. Two approaches have been employed to assess children with pre-existing conditions. The first approach has examined findings among specific groups of children represented in population-based, clinical, or atrisk samples. The second approach has assessed outcomes in samples of children with specific pre-existing psychological vulnerabilities.

\section{General Population, Clinical, and At-Risk Samples}

Studies of population-based samples have documented adverse outcomes in psychologically vulnerable children. For example, a large-scale population-based study of Hong Kong children revealed that those with special educational needs, chronic diseases, and/or acute health problems in the last 4 weeks and those whose parents had mental disorders and/or chronic diseases were more likely to experience emotional and behavioral problems during COVID19 school closure [32]. In their study of a large national sample of parents of Japanese children, Takahashi and Honda [22] found that children with neurodevelopmental disorders had the highest proportions of clinical-level emotional symptoms, conduct problems, hyperactivity/ inattention, and problems with peer relationships. Wright and colleagues [11•] found that maternally reported elevated internalizing symptoms in their children at 7 years of age predicted self-reported clinically significant depressive symptoms pre and post lockdown in the U.K. children they assessed. Internalizing and externalizing problems at age 7 years predicted both depression and behavior problems pre and post lockdown [11•].

Clinical and at-risk samples also have been assessed to ascertain the susceptibility of psychologically vulnerable children. Zijlmans and colleagues [33] compared psychiatric, pediatric, and general population samples of children in the Netherlands from April to May 2020 during COVID-19 lockdown. Children with pre-existing psychiatric disorders had worse emotional and social health than either those with chronic physical conditions or those in the general population [33]. In another study, conducted during the initial stage of COVID-19, Hawke and colleagues [34] assessed adolescents and young adults (14 to 28 years of age) participating in existing community and clinical cohort studies in Ontario, Canada. Mental health declined in both study settings, but contrary to the findings of Zijlmans and colleagues [33], the decline was greater in the community group [34]. In a study examining clinical notes on children in a large central Denmark catchment area, only a small proportion of children who were receiving psychiatric services in February and March 2020 experienced pandemic-related psychopathology [35]. Anxiety symptoms due to fear of COVID-19 were noted in children with anxiety, stress, and/or mood disorders while behavior symptoms increased in those with developmental disorders (e.g., autism, attention deficit hyperactivity disorder) likely due to disruption in routine and structures [35].

In a study of Welsh primary school students (aged 4 to 8 years at initial assessment before the pandemic and 5 to 10 years during lockdown), identified by their teachers as being at risk for mental health problems, Adegboye and colleagues [36] found high and increased levels of mental health problems during the pandemic lockdown. The change was most prominent in internalizing problems with increased generalized anxiety, panic and somatic symptoms, and school anxiety and decreased social and separation anxiety. There was no increase in externalizing problems in this sample which had high levels of externalizing problems at baseline [36].

\section{Samples of Children with Specific Pre-existing Conditions}

A developing literature has assessed samples of children with specific pre-existing conditions in the context of COVID-19. A number of these conditions are discussed below. 


\section{Special Education and Learning Disabilities}

Asbury and colleagues [37] queried U.K. parents of children with educational and other disabilities shortly after school closure. Parents reported that their children experienced loss (of, e.g., routine, support structures and network, participation of specialists), worry (e.g., for self, for others, in general), mood and behavior problems, difficulty understanding the pandemic, and challenges associated with remote learning [37]. Another study found that relative to pre-COVID-19 measures, a small sample of Spanish children with dyslexia experienced significantly greater emotional symptoms, conduct problems, and hyperactivity/inattention during quarantine, and a higher percentage of the children evidenced clinically significant problems across all three domains during quarantine [38].

\section{Attention Deficit Hyperactivity Disorder}

A sample of Chinese parents of school children with attention deficit hyperactivity disorder (ADHD) reported increased ADHD behaviors during COVID-19 school closure [39]. More than one-half of parents reported that their children's ability to focus and to maintain a routine declined and that they expressed anger more frequently, but more than one-half of parents also reported no change or improvement in other behaviors including neatness; ability to work quietly, to avoid interrupting adults, and to listen to instructions; and eating and sleeping behavior [39].

\section{Autism}

Because of their obsession with routine [40] and difficulty with the unexpected and with change and disruption [41], children with autism spectrum disorder are likely to experience heightened challenges during the pandemic [40, 41]. In a sample recruited from an Italian regional autism center, a majority of parents of children with autism spectrum disorder reported increased difficulty managing their children's daily activities and over one-third reported more frequent and more intense behavior problems in their children [41].

\section{Obsessive Compulsive Disorder}

Children with obsessive compulsive disorder (OCD) are thought to be especially vulnerable in the context of COVID19 because concerns about contagion and hygiene raised by the pandemic may aggravate OCD symptoms [42]. An investigation of Turkish children diagnosed with OCD—most of whom were in active treatment-revealed an increase in the severity of OCD symptoms in more than one-half of the participants during the pandemic [43]. The most common OCD symptoms in this sample-both before and during the pandemic-were contamination obsessions and cleaning compulsions [43]. A study conducted in Denmark assessed patient files in a group of children in clinical treatment for newly diagnosed OCD (clinical group) and surveyed a group identified through an OCD organization most of whom had completed treatment for OCD diagnosed years earlier (survey group) [44]. OCD, anxiety, and depression symptoms increased in both groups, especially the survey group [44].

\section{Early Life Stress}

A longitudinal study with pre-COVID-19 data on depression symptoms and stress in U.S. adolescents from Northern California revealed that severity of exposure to early life stress-from a variety of experiences-was associated with greater depression symptoms and with greater perceived stress in the initial weeks following COVID-19 restrictions [45]. Furthermore, rates of depression and stress during the pandemic were high with over $60 \%$ of participants meeting the clinical cut-off score for depression [45].

\section{Improved Outcomes}

Some children appear to improve in the context of the pandemic. For example, a U.K. survey found that some children, especially those with pre-pandemic difficulties, improved on measures of anxiety, depression, and/or wellbeing during the lockdown [46]. In the study by Asbury and colleagues [37], a substantial minority of parents of children with educational and other disabilities noted that their children experienced minimal or even positive reactions to the pandemic, especially children who had difficulty at school. A study examining predominantly Hispanic public charter students in grades five to eight found lower mental health symptom levels in the first several months after school closure relative to pre-pandemic levels in children with pre-pandemic mental health problems [47•]. This decrease was clinically significant for internalizing, externalizing, and total problems. Symptom improvement may have been due to increased time with family, improved sleep with more flexible schedules, reduced academic pressure, decreased stress from peer interactions, and the availability of school and social support in this community. It was unclear how long the benefits might continue [47•]. In a small Israeli sample of children in treatment for OCD, more participants evidenced improvement rather than worsening of symptoms and functioning, perhaps because their compulsive rituals were less obvious in the context of recommendations related to hygiene and/or 
because expectations related to school and social interactions were reduced [48].

As noted earlier, some types of anxiety (e.g., social anxiety, separation anxiety, performance anxiety) may have decreased in the context of home confinement because the usual pressures associated with school and social interactions were lessened. In the study of Welsh children by Adegboye and colleagues [36], generalized anxiety, panic and somatic symptoms, and school anxiety increased while social and separation anxiety decreased. Thus, children with social anxiety, social phobia, performance anxiety, and/or agoraphobia may experience temporary symptom reduction with school closure, physical distancing, and a decrease in daily stresses. Unfortunately, they are likely to fare poorly when re-exposed to their usual educational and social environments $[49,50]$.

Because they have established therapeutic relationships and resources, children who have been in treatment for pre-existing conditions may improve relative to other children-both those with and those without pre-existing psychological vulnerabilities. For example, a longitudinal study of a small sample comparing adolescents with early life stress (all of whom reported child maltreatment and met criteria for at least one anxiety and/or depressive disorder) and healthy adolescents found sizeable increases in depression and anxiety symptoms in healthy adolescents but not in those with early life stress 3 months after the onset of the pandemic [51]. Participants with early life stress had high but stable symptom scores when assessed before and during the pandemic and may have accessed pre-existing services and/or used medication generally not used by the healthy comparison group [51].

\section{Discussion}

Novel aspects of the COVID-19 pandemic are reflected in children's psychological reactions to the pandemic. While PTSD and PTSD symptoms are the most commonly studied child disaster outcomes, the experiences of many, if not most, children affected by the COVID-19 pandemic do not meet criteria for exposure to trauma as required for a diagnosis of PTSD. Depression, another common outcome in children exposed to disasters, has been widely reported in the child COVID-19 literature. The reported increase in suicidal ideation and behavior in the context of COVID-19 physical distancing and lockdown measures is of concern. Findings with respect to anxiety outcomes are less clear, suggesting the importance of distinguishing various sources and types of anxiety because some children may experience symptom relief with decreased social, academic, and other demands occasioned by school closure and other physical distancing measures. Unfortunately, a rebound in symptoms is likely once children return to school and their normal routines. Perhaps reflecting changes in socialization and access to substances during home confinement, the COVID-19 substance use findings suggest an inadvertent and unexpected positive effect in substance use behaviors in some youth.

Like child disaster mental health studies in general, the COVID-19 literature affirms that the vulnerable are vulnerable-children with pre-existing social, family, health, and mental health vulnerabilities are at heightened risk for adverse outcome. While not fully understood, some children with pre-existing emotional problems appear to fare better during physical distancing and home confinement than children without, perhaps because they have more time with parents and family; because stress associated with school and in other arenas where performance is expected and/or monitored is reduced; and/or because they have established services, therapeutic relationships, and resources though these services, relationships, and resources may be interrupted during the pandemic.

While space constraints preclude an examination of numerous potential correlates of outcome, factors likely to influence children's reactions include those identified in the child disaster literature such as exposure, demographics, preexisting conditions and vulnerabilities, and family and social factors. In the context of COVID-19, public health measures aimed at disease containment are likely to have exerted dramatic effects on children's reactions through, for example, changes in family relationships and dynamics, altered daily routines and lifestyle behaviors (e.g., diet, sleep, physical activity, screen time), diminished academic and social pressure, and access to health and mental health services.

Worldwide concern about COVID-19 has stimulated a veritable explosion of research including numerous investigations of children's psychological reactions. Many studies were fielded early, often taking advantage of relatively recently employed online survey design. Studies have assessed numerous samples using diverse research methods, designs, and instruments to examine a variety of outcomes at various times across the course of the pandemic. Much of the research has used cross-sectional design, retrospective reporting, and non-validated instruments to assess non-representative samples which limit the generalizability and interpretation of the findings. Some studies took advantage of pre-existing data and routine surveillance to document changes in children's status, but most studies relied on retrospective reporting by children themselves and/or their parents. Few longitudinal studies have been conducted and follow-up assessments have been rare, thereby precluding an investigation of the trajectories of response or the long-term implications of the pandemic on children's mental health and functioning. The clinical significance of children's reactions was not routinely assessed. Future research should consider using robust designs with attention to characteristics of the samples, sampling 
approaches, informants, and validated assessment tools as well as multi-variate analysis to examine the differential and interactive effects of the pandemic with other adverse child experiences and psychosocial and mental health variables.

\section{Conclusions}

A great deal of research supporting general concerns about the effects of the COVID-19 pandemic on children's psychological status has been published in a short period of time. Clearly, the pandemic has been harmful to children around the globe. While many of the psychological consequences parallel those seen in other disasters, novel features of this crisis, especially the associated public health measures that closed schools and confined children to home for extended periods, have produced results not commonly explored in disasters. The specific effects of these containment measures warrant consideration in future research. A lack of methodological rigor in many studies, along with the use of diverse samples and instruments and the variation in the timing of data collection, make it difficult to interpret some results which may not generalize across populations. Thus, findings to date should be considered preliminary until more rigorous research has been conducted. Moreover, the preponderance of the literature has focused on the adverse consequences associated with the COVID-19 experience. Additional research is needed to examine adaptive coping and resilience in children in this context, and research is needed to explore the challenges and effects of reentry into in-person environments when schools and other facilities reopen and post pandemic. Given the persistence of the COVID-19 pandemic, and the likelihood of future pandemics, it is imperative that research fully explores the psychological consequences in children and guides the mental health response.

Acknowledgements The editors would like to thank Dr. Andres J. Pumariega for taking the time to review this manuscript.

\section{Compliance with Ethical Standards}

Conflict of Interest The author declares no competing interests.

Human and Animal Rights and Informed Consent This article does not contain any studies with human or animal subjects performed by any of the authors.

\section{References}

Papers of particular interest, published recently, have been highlighted as:

- Of importance

$\bullet$ Of major importance
1. Morganstein JC, Fullerton CS, Ursano RJ, Donato D, Holloway HC. Pandemics: health care emergencies. In: Ursano R, Fullerton CS, Weisaeth L, Raphael B, editors. Textbook of disaster psychiatry, 2nd Ed. Cambridge: Cambridge University Press; 2017. p. 270-84. https://doi.org/10.1017/9781316481424.019.

2. Ashikkali L, Carroll W, Johnson C. The indirect impact of COVID19 on child health. Paediatr Child Health. 2020;30(12):430-7. https://doi.org/10.1016/j.paed.2020.09.004.

3. Aleebrahim-Dehkordi E, Soveyzi F, Deravi N, Rabbani Z, Saghazadeh A, Rezaei N. Human coronaviruses SARS-CoV, MERS-CoV, and SARS-CoV-2 in children. J Pediatr Nurs. 2021;56:70-9. https://doi. org/10.1016/j.pedn.2020.10.020.

4. Viner RM, Mytton OT, Bonell C, Melendez-Torres GJ, Ward J, Hudson L, et al. Susceptibility to SARS-CoV-2 infection among children and adolescents compared with adults. A systematic review and meta-analysis. JAMA Pediatr. 2021;175(2):14356. https://doi.org/10.1001/jamapediatrics.2020.4573.

5. Hillis SD, Unwin HJT, Chen Y, Cluver L, Sherr L, Goldman PS et al. Global minimum estimates of children affected by COVID19-associated orphanhood and deaths of caregivers: a modelling study Lancet 2021;398:391-402. https://doi.org/10.1016/S01406736(21)01253-8

6. Liu D, Liu W, Rodriguez M, Zhang J, Zhang F. The mental health impacts of COVID-19 on pediatric patients following recovery. Front Psychol. 2021;29:628-707. https://doi.org/10.3389/fpsyg. 2021.628707.

7. Qin Z, Shi L, Xue Y, Lin H, Zhang J, Liang P, et al. Prevalence and risk factors associated with self-reported psychological distress among children and adolescents during the COVID-19 pandemic in China. JAMA Netw Open. 2021;4(1): e2035487. https://doi.org/10.1001/jamanetworkopen.2020.35487.

8. Vizard T, Sadler K, Ford T, Newlove-Delgado T, McManus S, Marcheselli F, et al. Mental health of children and young people in England, 2020. Wave 1 follow up to the 2017 survey. NHS DigitalHealth and Social Care Information Centre. 2020. https://files.digital. nhs.uk/CB/C41981/mhcyp_2020_rep.pdf. Accessed 2 August 2021.

9. Mallik CI, Radwan RB. Impact of lockdown due to COVID-19 pandemic in changes of prevalence of predictive psychiatric disorders among children and adolescents in Bangladesh. Asian J Psychiatry. 2021;56: 102254. https://doi.org/10.1016/j.ajp.2021.102554.

10. Sayed MH, Hegazi MA, El-Baz MS, Alahmadi TS, Zubairi NA, Altuwiriqi MA, et al. COVID-19 related posttraumatic stress disorder in children and adolescents in Saudi Arabia. PLoS ONE. 2021;16(8): e0255440. https://doi.org/10.1371/journal.pone. 0255440 .

11. Wright N, Hill J, Sharp H, Pickles A. Interplay between longterm vulnerability and new risk: young adolescent and maternal mental health immediately before and during the COVID-19 pandemic. JCPP Adv. 2021; May 18. https://doi.org/10.1111/ jcv2.12008. This longitudinal population-based investigation in the U.K. reported increased symptoms of disruptive behavior in 11- to 12-year-old children, especially those without prior externalizing symptoms, three months after the implementation of social distancing measures relative to symptoms assessed immediately before the lockdown.

12. Ma Z, Idris S, Zhang Y, Zewen L, Wali A, Ji Y, et al. The impact of COVID-19 pandemic outbreak on education and mental health of Chinese children aged 7-15 years: an online survey. BMC Pediatr. 2021;21:95. https://doi.org/10.1186/s12887-021-02550-1.

13. Yue J, Zang X, Le Y, An Y. Anxiety, depression and PTSD among children and their parent during 2019 novel coronavirus disease (COVID-19) outbreak in China. Curr Psychol. 2020; November 14:1-8. https://doi.org/10.1007/s12144-020-01191-4.

14. Nearchou F, Flinn C, Niland R, Subramaniam SS, Hennessy E. Exploring the impact of COVID-19 on mental health outcomes in children and adolescents: a systematic review. Int $\mathbf{J}$ 
Environ Res Public Health. 2020;17:8479. https://doi.org/10. 3390/ijerph17228479.

15.•• Racine N, McArthur BA, Cooke JE, Eirich R, Zhu J, Madigan S. Global prevalence of depressive and anxiety symptoms in children and adolescents during COVID-19. A meta-analysis. JAMA Pediatr. 2021; August 9. https://doi.org/10.1001/jamapediatrics. 2021.2482. This meta-analysis of general population studies found clinically-elevated depression and anxiety symptom rates of $25.2 \%$ and $20.5 \%$, respectively, in children during the pandemic. These rates are approximately twice the rates in pre-pandemic research.

16. Barendse MEA, Flannery J, Cavanagh C, Aristizabal M, Becker $\mathrm{SP}$, Berger E, et al. Longitudinal change in adolescent depression and anxiety symptoms from before to during the COVID19 pandemic: a collaborative of 12 samples from 3 countries. PsyArXiv. 2021; February 3. https://doi.org/10.31234/osf.io/ hn7us. This study, which analyzed composite data from 12 longitudinal investigations that conducted at least one assessment prior to the pandemic, found a significant increase in depression symptoms and no change in anxiety symptoms in children during the pandemic. The authors speculated that the lack of change in anxiety symptoms overall may have obscured possible changes in some types of anxiety. For example, social anxiety may have declined with pandemicrelated physical distancing measures because of a decrease in social interactions and social pressure.

17.•• Yard E, Radhakrishnan L, Ballesteros MF, Sheppard M, Gates A, Stein $\mathrm{Z}$ et al. Emergency department visits for suspected suicide attempts among persons aged 12-25 years before and during the COVID-19 pandemic - United States, January 2019-May 2021 MMWR 70 24:888-894. https://doi.org/10.15585/mmwr.mm702 $4 \mathrm{e} 1$. This study revealed an initial decrease in ED visits for suspected suicide attempts among adolescents during the spring of 2020 in comparison to visits in the spring of 2019, but ED visits for mental health issues and for suspected suicide attempts increased by early May 2020 especially in girls.

18. Hill RM, Rufino K, Kurian S, Saxena J, Saxena K, Williams L. Suicide ideation and attempts in a pediatric emergency department before and during COVID-19. Pediatrics. 2021;147(3): e2020029280. https://doi.org/10.1542/peds.2020-029280.

19. Pieh C, Plener PL, Probst T, Dale R, Humer E. Assessment of mental health of high school students during social distancing and remote schooling during the COVID-19 pandemic in Austria. JAMA Netw Open. 2021;4(6): e2114866. https://doi.org/ 10.1001/jamanetworkopen.2021.14866.

20. Zhang L, Zhang D, Fang J, Wan Y, Tao F, Sun Y. Assessment of mental health of Chinese primary school students before and after school closing and opening during the COVID-19 pandemic. JAMA Netw Open. 2020;3(9): e2021482. https://doi.org/ 10.1001/jamanetworkopen.2020.21482.

21. Isumi A, Doi S, Yamaoka Y, Takahashi K, Fujiwara T. Do suicide rates in children and adolescents change during school closure in Japan? The acute effect of the first wave of COVID-19 pandemic on child and adolescent mental health. Child Abuse Negl. 2020;110: 104680. https://doi.org/10.1016/j.chiabu.2020.104680.

22. Takahashi F, Honda H. Prevalence of clinical-level emotional, behavioral problems in schoolchildren during the coronavirus disease, 2019 pandemic in Japan: a prospective cohort study JCPP Adv 2021 e12007. https://doi.org/10.1111/jcv2.12007.

23. Degenhardt L, Stockings E, Patton G, Hall WD, Lynskey M. The increasing global health priority of substance use in young people. Lancet Psychiatry. 2016;3:251-64. https://doi.org/10. 1016/S2215-0366(15)00508-8.

24. Thorisdottir IE, Asgeirsdottir BB, Kristjansson AL, Valdimarsdottir HB, Jonsdottir Tolgyes EM, Sigfusson J et al. Depressive symptoms, mental wellbeing, and substance use among adolescents before and during the COVID-19 pandemic in Iceland: a longitudinal, population-based study Lancet Psychiatry 2021;8(8):663-672. https://doi.org/10. 1016/S2215-0366(21)00156-5.This national longitudinal study of Icelandic adolescents revealed decreased cigarette smoking, e-cigarette use, and alcohol intoxication in 15- to 18-year-old participants during the pandemic relative to rates in 2016 and 2018.

25. Chaffee BW, Cheng J, Couch ET, Hoeft KS, Halpern-Felsher B Adolescents' substance use and physical activity before and during the COVID-19 pandemic. JAMA Pediatr. 2021;175(7):71522. https://doi.org/10.1001/jamapediatrics.2021.0541.

26. Gaiha SM, Lempert LK, Halpern-Felsher B. Underage youth and young adult e-cigarette use and access before and during the coronavirus disease 2019 pandemic. JAMA Netw Open. 2020;3(12): e2027572. https://doi.org/10.1001/jamanetworkopen. 2020.27572

27. Dumas TM, Ellis W, Litt DM. What does adolescent substance use look like during the COVID-19 pandemic? Examining changes in frequency, social contexts, and pandemic-related predictors. J Adolesc Health. 2020;67:354-61. https://doi.org/ 10.1016/j.jadohealth.2020.06.018.

28. Pagoto SL, Conroy DE. Revitalizing adolescent health behavior after the COVID-19 pandemic. JAMA Pediatr. 2021;175(7):6779. https://doi.org/10.1001/jamapediatrics.2021.0547.

29. Liu S, Liu Y, Liu Y. Somatic symptoms and concern regarding COVID-19 among Chinese college and primary school students: a cross-sectional survey. Psychiatry Res. 2020;289: 113070. https://doi.org/10.1016/j.psychres.2020.113070.

30. Malas N, Ortiz-Aguayo R, Giles L, Ibeziako P. Pediatric somatic symptom disorders. Curr Psychiatry Rep. 2017;19:11. https:// doi.org/10.1007/s11920.017.0760.3.

31.•• Patrick SW, Henkhaus LE, Zickafoose JS, Lovell K, Halvorson A, Loch $\mathrm{S}$ et al. Well-being of parents and children during the COVID19 pandemic: a national survey Pediatrics 2020;146:4. https://doi. org/10.1542/peds.2020.016824. This study polled a representative sample of parents in the U.S. in June 2020. Almost 15\% reported worsening of their children's behavioral health and approximately $10 \%$ reported that their children's behavioral health had deteriorated since March 2020, but most parents reported no change in their children's behavioral, mental, and physical health.

32. Tso WWY, Wong RS, Tung KTS, Rao N, Fu KW, Yam JCS, et al. Vulnerability and resilience in children during the COVID19 pandemic. Eur Child Adolesc Psychiatry. 2020; November 17. https://doi.org/10.1007/s00787-020-01680-8.

33. Zijlmans J, Teela L, van Ewijk H, Klip H, van der Mheen M, Ruisch H, et al. Mental and social health of children and adolescents with pre-existing mental or somatic problems during the COVID-19 pandemic lockdown. Front Psychiatry. 2021;12. https://doi.org/10.3389/fpsyt.2021.692853.

34. Hawke LD, Barbic SP, Voineskos A, Szatmari P, Cleverley K, Hayes E, et al. Impacts of COVID-19 on youth mental health, substance use, and well-being: a rapid survey of clinical and community samples. Can J Psychiatry. 2020;65(10):701-9. https://doi.org/10.1177/0706743720940562.

35. Jefsen $\mathrm{OH}$, Rohde $\mathrm{C}, \mathrm{N} \varnothing$ rremark B, Østergaard SD. Editorial perspective: COVID-19 pandemic-related psychopathology in children and adolescents with mental illness. J Child Psychol Psychiatry. 2021;62(6):798-800. https://doi.org/10.1111/jcpp. 13292.

36. Adegboye D, Williams F, Collishaw S, Shelton K, Langley K, Hobson C, et al. Understanding why the COVID-19 pandemicrelated lockdown increases mental health difficulties in vulnerable young children. JCPP Adv. 2021;1-8. https://doi.org/10. 1111/jcv2.12005. 
37. Asbury K, Fox L, Deniz E, Code A, Toseeb U. How is COVID-19 affecting the mental health of children with special educational needs and disabilities and their families? J Autism Dev Dis. 2021;51:1772-80. https://doi.org/10.1007/s10803-020-04577-2.

38. Soriano-Ferrer M, Morte-Soriano MR, Begeny J, PiedraMartinez E. Psychoeducational challenges in Spanish children with dyslexia and their parents' stress during the COVID-19 pandemic. Front Psychol. 2021;12: 648000. https://doi.org/10. 3389/fpsyg.2021.648000.

39. Zhang J, Shuai L, Yu H, Wang Z, Qiu M, Lu L, et al. Acute stress, behavioural symptoms and mood states among schoolage children with attention-deficit/hyperactive disorder during the COVID-19 outbreak. Asian J Psychiatry. 2020;51: 102077. https://doi.org/10.1016/j.ajp.2020.102077.

40. Eshraghi AA, Li C, Alessandri M, Messinger DS, Eshraghi RS, Mittal R, Armstrong FD. COVID-19: overcoming the challenges faced by individuals with autism in their families. Lancet Psychiatry. 2020;7(6):481-3. https://doi.org/10.1016/s2215-0366(20) 30197-8.

41. Colizzi M, Sironi E, Antonini F, Ciceri ML, Bovo C, Zoccante L. Psychosocial and behavioral impact of COVID-19 in autism spectrum disorder: an online parent survey Brain Sci 2020;10:341. https://doi. org/10.3390/brainsci10060341.

42. Singh S, Roy D, Sinha K, Parveen S, Sharma G, Joshi G. Impact of COVID-19 and lockdown on mental health of children and adolescents: a narrative review with recommendations. Psychiatry Res. 2020;293: 113429. https://doi.org/10.1016/j.psychres.2020.113429.

43. Tanir Y, Karayagmurlu A, Kaya İ, Kaynar TB, Türkmen G, Dambasan BN, et al. Exacerbation of obsessive compulsive disorder symptoms in children and adolescents during COVID-19 pandemic. Psychiatry Res. 2020;293: 113363. https://doi.org/10. 1016/j.psychres.2020.113363.

44. Nissen JB, Højgaard DRMA, Thomsen PH. The immediate effect of COVID-19 pandemic on children and adolescents with obsessive compulsive disorder. BMC Psychiatry. 2020;20:511. https://doi.org/10.1186/s12888-020-02905-5.

45. Gotlib IH, Borchers LR, Chahal R, Gifuni AJ, Teresi GI, Ho TC. Early life stress predicts depressive symptoms in adolescents during the COVID-19 pandemic: the mediating role of perceived stress. Front Psychol. 2021;11: 603748. https://doi.org/10.3389/ fpsyg.2020.603748.
46. Widnall E, Winstone L, Mars B, Haworth C, Kidger J. Young people's mental health during the COVID-19 pandemic. Initial findings from a secondary school survey study in South West England. NIHR School for Public Health Research, 2020, August. https:// sphr.nihr.ac.uk/research/young-peoples-mental-health-during-thecovid-19-pandemic/. Accessed 2 August 2021.

47. Penner F, Hernadez J, Ortiz Sharp C. Change in youth mental health during the COVID-19 pandemic in a majority Hispanic/LatinX US sample J Am Acad Child Adolesc Psychiatry 2021;60(4):513-523. https://doi.org/10.1016/j.jaac.2020.12.027. This study examining a sample of predominantly Hispanic public charter students in grades five to eight found lower mental health symptom levels in the first several months after school closure relative to pre-pandemic levels in children with pre-pandemic mental health problems. The decrease was clinically significant for internalizing, externalizing, and total problems. Symptom improvement may have been due to increased time with family, improved sleep, reduced academic and social pressure, and the availability of school and social support in this community.

48. Schwartz-Lifshitz M, Basel D, Lang C, Hertz-Palmor N, Dekel I, Zohar J, Gothelf D. Obsessive compulsive symptoms severity among children and adolescents during COVID-19 first wave in Israel. J Obsessive Compuls Relat Disord. 2021;28: 100610. https://doi.org/10.1016/j.jocrd.2020.100610.

49. Courtney D, Watson P, Battaglia M, Mulsant BH, Szatmari P. COVID-19 impacts on child and youth anxiety and depression: challenges and opportunities. Can J Psychiatry. 2020;65(10):68891. https://doi.org/10.1177/0706743720935646.

50. Pumariega AJ. Editorial: Mental health and schools: has the time arrived? J Am Acad Child Adolesc Psychiatry. 2021, in press. Doi:https://doi.org/10.1016/j.jaac.2021.05.001.

51. Cohen ZP, Cosgrove KT, DeVille DC, Akeman E, Singh MK, White E, et al. The impact of COVID-19 on adolescent mental health: preliminary findings from a longitudinal sample of healthy and at-risk adolescents. Front Pediatr. 2021; June 8. Doi: https:// doi.org/10.3389/fped.2021.622608.

Publisher's Note Springer Nature remains neutral with regard to jurisdictional claims in published maps and institutional affiliations. 